\title{
Prolactin increases SMN expression and survival in a mouse model of severe spinal muscular atrophy via the STAT5 pathway
}

\author{
Faraz Farooq,1,2 Francisco Abadía Molina, ${ }^{3}$ Jeremiah Hadwen,1,2 Duncan MacKenzie,1,2
} Luke Witherspoon, 1,2 Matthew Osmond,1 Martin Holcik, ${ }^{1,2}$ and Alex MacKenzie1,2

${ }^{1}$ University of Ottawa, Ottawa, Canada. ${ }^{2}$ Apoptosis Research Center, CHEO Research Institute, CHEO, Ottawa, Canada. ${ }^{3}$ Biología Celular, Universidad de Granada, Granada, Spain.

\begin{abstract}
Spinal muscular atrophy (SMA) is an autosomal recessive neurodegenerative disease that is characterized by the loss of motor neurons, resulting in progressive muscle atrophy. It is caused by the loss of functional survival motor neuron (SMN) protein due to mutations or deletion in the SMN1 gene. A potential treatment strategy for SMA is to upregulate levels of SMN protein. Several agents that activate STAT5 in human and mouse cell lines enhance SMN expression from the SMN2 gene and can compensate, at least in part, for the loss of production of a functional protein from SMN1. Here, we have shown that prolactin (PRL) increases SMN levels via activation of the STAT5 pathway. PRL increased SMN mRNA and protein levels in cultured human and mouse neuronal cells. Administration of STAT5-specific siRNA blocked the effects of PRL, indicating that the PRL-induced transcriptional upregulation of the SMN-encoding gene was mediated by activation of STAT5. Furthermore, systemic administration of PRL to WT mice induced SMN expression in the brain and spinal cord. Critically, PRL treatment increased SMN levels, improved motor function, and enhanced survival in a mouse model of severe SMA. Our results confirm earlier work suggesting STAT5 pathway activators as potential therapeutic compounds for the treatment of SMA and identify PRL as one such promising agent.
\end{abstract}

\section{Introduction}

Autosomal recessive spinal muscular atrophy (SMA) is a leading inherited cause of infant death worldwide. It is characterized by the loss of motor neurons from the anterior horn of spinal cord and attendant paresis leading to respiratory insufficiency (1). The incidence of SMA is one in 10,000 live births, with a carrier frequency of approximately 1:35 to 1:50 (2). Based on disease onset and severity, SMA is broadly classified into 4 categories: type I, the most common and severe form in which there is a profound weakness by 6 months, often resulting in death by the first 2 years of life; types II and III, which are milder forms; and type IV, characterized by mild proximal weakness at adulthood and normal longevity.

Low levels of functional survival motor neuron (SMN) protein due to mutations or deletion of the $\operatorname{SMN1}$ gene cause $\operatorname{SMA}(3,4)$. $\mathrm{SMN}$ is a ubiquitously expressed $294-$ amino acid protein evolutionarily conserved, which through a myriad of interactions is required for subcellular RNA localization and processing (4-12). Chief among these is a complex with Gemins, central to biogenesis of spliceosomal small nuclear ribonucleoproteins (snRNPs) a major component of pre-mRNA splicing machinery (13). SMN has other functions as well in the assembly, metabolism, and transport of other ribonucleoproteins $(8,10,12)$. Due to an evolutionarily recent duplication event on chromosome $5 \mathrm{q} 13$, humans uniquely have 2 nearly identical SMN genes, SMN1 and SMN2 $(14,15)$. The SMN1 gene is the primary source of full-length functional SMN protein; its loss or mutation is both necessary and sufficient for SMA (3). There are 5 nucleotides that distinguish SMN1 and SMN2 coding regions (3); none are translationally significant, although

Conflict of interest: The authors have declared that no conflict of interest exists. Citation for this article: J Clin Invest. 2011;121(8):3042-3050. doi:10.1172/JCI46276. the $\mathrm{C}$ to $\mathrm{T}$ transition at position 6 of exon 7 results in SMN2 mRNA being alternatively spliced, producing only $5 \%-10 \%$ of the full-length functional SMN protein $(3,16,17)$.

All SMA patients have at least one copy of the SMN2 gene, which produces low amounts of functional SMN protein. Complete absence of $S M N$ genes is embryonically lethal in both humans and mice (18-20). Individuals with SMA have a variable number of SMN2 genes; the greater the SMN2 gene copy number, the milder the disease severity $(21,22)$. Presently, there is no effective therapy for SMA. A potential treatment strategy for SMA is to upregulate levels of SMN protein originating from the SMN2 gene, compensating in part for the absence of a functional SMN1 gene.

A number of small molecules have been proposed as SMN2 inducers; among them are sodium valproate, trichostatin A (TSA), and aclarubicin, which have all been suggested to activate STAT5 in SMA-like mouse embryonic fibroblasts and human SMN2-transfected NSC34 cells (23). STAT5 is a member of the Signal Transducers and Activator of Transcription proteins family, which controls diverse cellular responses such as cell growth, differentiation, and apoptosis through modification of gene expression of cytokine inducible genes $(24,25)$. STATs are direct targets of JAK, which is activated by the binding of ligand to specific cytokine receptors. Prolactin (PRL) is a 199-amino acid 23-kDa polypeptide hormone that binds to PRL receptor (PRLR) and activates the JAK2/STAT5 pathway $(25,26)$. PRLRs are present in the metazoan central nervous system, including motor neurons $(25,27)$, and the protein is blood brain permeant, enticing us to explore its impact on SMN2.

We show here a PRL-conferred SMN increase in both neuronal cells and WT mice and present evidence for a STAT5 pathway activation of SMN transcription underlying this increase in functional SMN protein. Importantly, we show that PRL also increases SMN levels and improves motor function and survival 
A

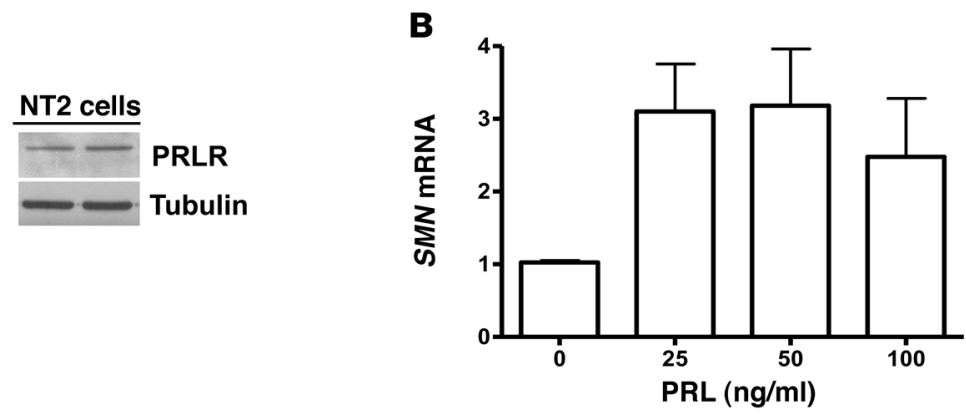

D

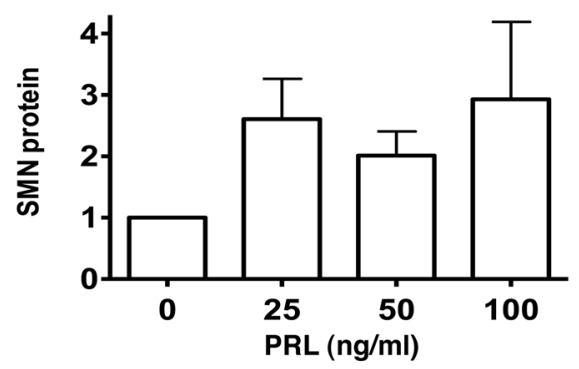

E

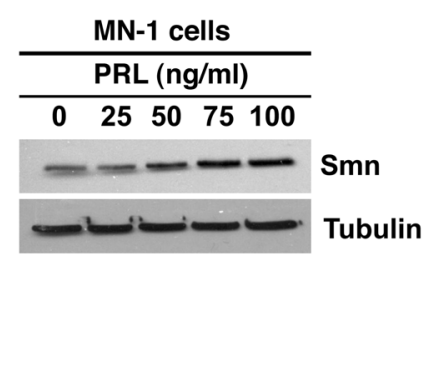

C

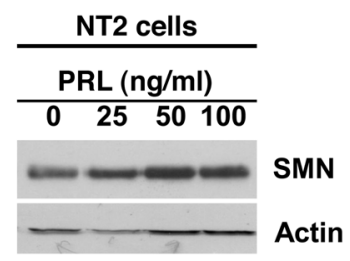

$\mathbf{F}$

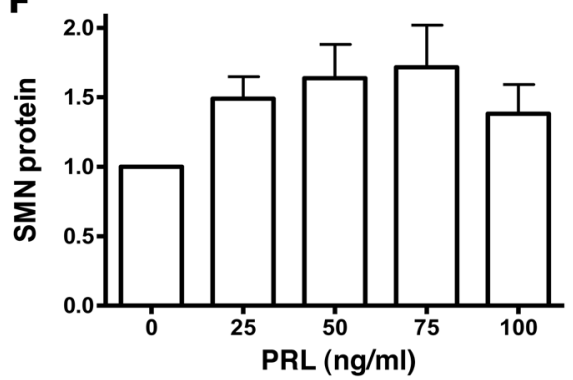

Figure 1

PRL treatment upregulates SMN mRNA and protein in vitro. NT2 or MN-1 cells were treated with PRL $(0,25,50$, and $100 \mathrm{ng} / \mathrm{ml})$ and then harvested at indicated intervals for RT-PCR or Western blot analyses. (A) Presence of PRLR in NT2 cells. (B) Quantification of SMN mRNA relative to $\beta$-actin in NT2 cells after PRL treatment (fold induction; the ratio at 0 hour was set as 1 ). Mean + SD of 3 independent experiments. (C) Representative Western blot showing effect of PRL on SMN protein in NT2 cells. (D) Densitometric quantification of SMN relative to $\beta$-actin is shown for NT2 cells. Mean + SD of 3 independent experiments. (E) Representative Western blot showing effect of PRL on SMN protein in MN-1 cells. (F) Densitometric quantifications of Smn relative to tubulin are shown for MN-1 cells. Mean + SD of 3 independent experiments.

in a severe SMA mouse model. Our results confirm earlier work suggesting STAT5 pathway activators as potential therapeutic compounds for the treatment of SMA and identify PRL as one such promising agent.

\section{Results}

PRL treatment upregulates SMN gene expression in vitro. In order to explore a role for PRL in the regulation of the SMN gene in vitro, the expression of PRLR in human neuron-committed teratocarcinoma (NT2) cells was first confirmed (Figure 1A). The cells were next treated with a range of PRL doses $(25-100 \mathrm{ng} / \mathrm{ml})$ for 48 hours. SMN transcript levels were found to be increased significantly (3- to 4-fold) in NT2 cells upon treatment with all PRL doses (Figure 1B). NT2 or motor neuron-derived (MN-1) cells were then treated with similar PRL doses for 48 hours and harvested for Western blot analysis. All PRL doses tested led to a significant increase in SMN protein levels in both NT2 and MN-1 cells (Figure 1, $\mathrm{C}-\mathrm{F})$. Taken together, these results demonstrate that PRL treatment causes an increase in SMN steady-state mRNA and protein levels in both human and mice neuronal cell lines.

Activation of STAT5 pathway causes upregulation of SMN gene and is responsible for $P R L$-conferred SMN protein increase. Since the STAT5 pathway has been implicated in the regulation of the SMN gene (23) and given that PRL is a known activator of the pathway, we confirmed the previously published results that PRL increases total STAT5 levels and also activates the STAT5 pathway by phosphorylating STAT5 protein (Figure 2A). To further investigate a potential STAT5 role in SMN gene regulation, NT2 cells were treated with aurintricarboxylic acid (ATA; known STAT5 activator that mimics PRL activity; ref. 28) for 48 hours. Treatment with ATA led to a significant induction in SMN transcript levels similar to that seen with PRL treatment (Figure 2B). To further confirm the role of the STAT5 pathway in PRL-induced increase in SMN protein, NT2 cells were transfected with STAT5-specific siRNA or control siRNA for 24 hours and then treated with PRL for 48 hours. siRNA-mediated abrogation of STAT5 expression completely blocked the PRLinduced increase in SMN protein (Figure 2, C and D). The results when viewed in aggregate strongly implicate the STAT5 pathway in the PRL-induced increase of SMN levels.

PRL treatment upregulates SMN protein level in WT mice. In order to both confirm that PRL-mediated SMN induction extends to the in vivo setting and to begin to optimize the dose for PRL treatment, CD-1 mice were given daily i.p. PRL injections for 5 days over a range of doses. Brain and spinal cord samples were isolated for Western blot analysis. PRL treatment increased SMN protein levels in a dose-dependent manner in brain (Figure 3, A and B) and at higher doses in spinal cord samples (Figure 3, C and D).

PRL treatment upregulates SMN $m R N A$ and protein levels in an SMA mouse model. In order to explore the impact of PRL-induced SMN upregulation on SMA, genetically faithful transgenic $\left(\mathrm{mSmn} \mathrm{n}^{-1-}\right.$; $\left.S M N 2^{+/+}, S M N 2 * \Delta 7^{+/+}\right)$SMA mice $(\operatorname{SMA} \Delta 7)(29)$ were given $2.5 \mathrm{mg} / \mathrm{kg}$ PRL i.p. injections every day from P1 until P6. Mice were euthanized 24 hours after the last treatment. Brain and spinal cord samples were then harvested for RT-PCR and Western blot analysis. Importantly, PRL treatment was observed to significantly increase SMN2-derived full-length SMN transcript (Figure 4, $\mathrm{A}$ and $\mathrm{B}$ ) as well as protein levels in both brain and spinal cord samples when compared with vehicle-treated mice (Figure 4, C-F, and Supplemental Figure 1; supplemental material available online with this article; doi:10.1172/JCI46276DS1). 
A

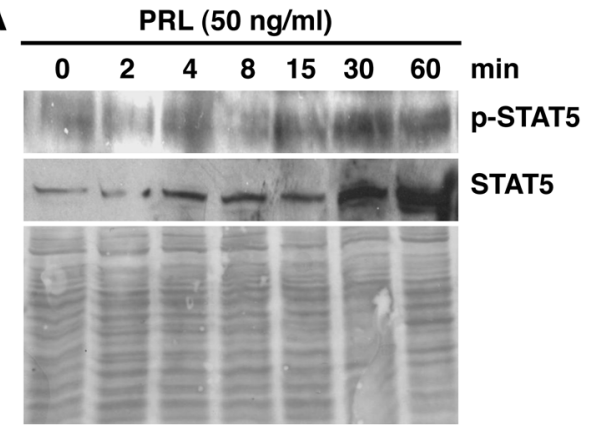

C

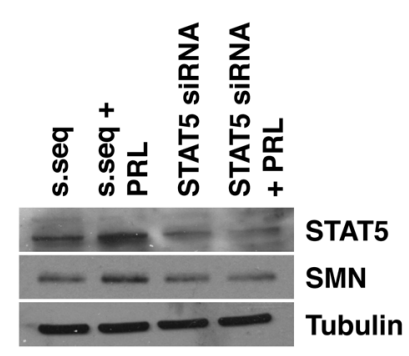

B

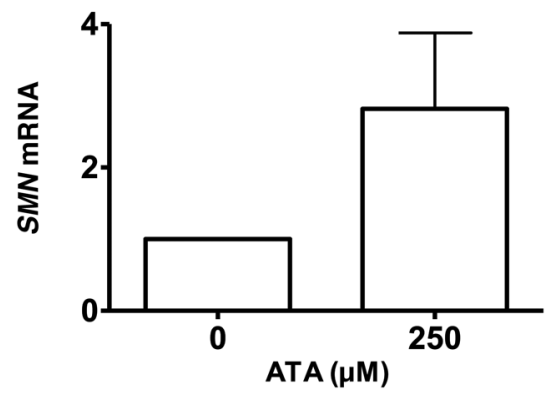

D

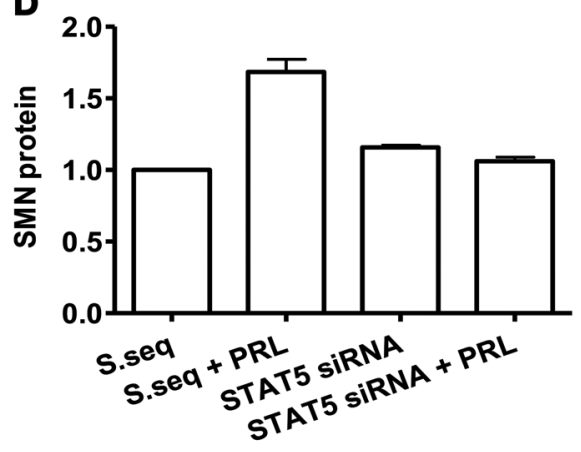

\section{Figure 2}

PRL increases SMN expression via STAT5 pathway. (A) Representative Western blot showing activation of STAT5 pathway upon PRL treatment in NT2 cells. (B) ATA (STAT5 pathway activator) treatment upregulates SMN mRNA in vitro. NT2 cells were treated with ATA $(250 \mu \mathrm{M})$ and then harvested after 48 hours for RT-PCR. Quantification of SMN mRNA relative to $\beta$-actin in NT2 cells after ATA treatment (fold induction; the ratio at 0 hours was set as 1). Mean + SD of 3 independent experiments is shown. (C) The siRNA knockdown of STAT5 protein attenuates PRL-induced increase in SMN protein. Representative Western blots showing both STAT5 knockdown and its effect on PRL-induced increase in SMN protein. s. seq., scrambled sequence. (D) Densitometric quantification of SMN relative to tubulin is shown. Mean + SD of 3 independent experiments is shown.
PRL treatment upregulates SMN expression within motor neurons and endothelial cells in an SMA mouse model. To check the expression of SMN protein within motor neurons after treatment with PRL, SMA $\Delta 7$ mice were given a daily $2.5 \mathrm{mg} / \mathrm{kg}$ PRL i.p. dose from P1 till P6. Mice were euthanized 24 hours after the last treatment. Brain and spinal cord samples were harvested for immunohistochemistry analysis. Motor neurons were labeled with HB9 antibody (motor neuron marker). PRL treatment increased SMN protein levels significantly in motor neurons of both brain and spinal cord samples when compared with vehicle-treated mice (Figure 5, A-F). Also SMN levels were significantly higher in endothelial cells among treatment groups (Figure 5, G and $\mathrm{H}$ ).

PRL treatment improves disease phenotype of SMA mouse model. The impact of PRL-induced increase in SMN levels on disease phenotype in SMA $\Delta 7$ mice was next examined. The SMA $\Delta 7$ mice have severely impaired righting time and muscle weakness detectable by P5. They are also significantly underweight compared with heterozygous and WT littermates, with a median survival of 13 days. In order to assess the impact of PRL on the phenotype of the disease, SMA $\Delta 7$ mice (P1) were given daily PRL or vehicle i.p. injections and their weight, motor function, and longevity assessed. SMA $\Delta 7$ mice treated with PRL showed a considerably slower weight loss, dramatic improvement of motor function (as assessed by righting time), and significant extension of survival (median survival of 21 days) as compared with vehicle-treated SMA $\Delta 7$ mice (median survival of 14 days; Figure 6, A-C, respectively, and Supplemental Videos 1-3).

PRL treatment upregulates SMN protein level in an SMA mouse model. In order to investigate whether SMN is upregulated throughout the life span, SMA $\Delta 7$ mice were given daily $2.5 \mathrm{mg} / \mathrm{kg}$ PRL i.p. doses from P1 till death (P23). Brain, spinal cord, and muscle samples were harvested for Western blot analysis. SMN protein levels in brain and spinal cord tissues were found to be significantly increased at the time of death (Figure 7, A-D), with smaller increases observed in muscle (Figure 7, E and F).

\section{Discussion}

A potential treatment strategy for SMA is the upregulation of SMN protein originating from $S M N 2$, a gene that is present in all affected SMA individuals, compensating in part for the absence of a functional SMN1 (3). One of a number of pathways that increase SMN is STAT5 (23), and one of the more potent known inducers of STAT5 is PRL. The facts that PRL passes the blood brain barrier (BBB) and that its receptor is expressed throughout the central nervous system $(26,27)$ increase its potential as an SMA therapeutic.

We document here a PRL-mediated increase in both SMN mRNA and protein levels in the human neuronal cell line and murine motor neuron MN-1 cells. We also show an associated rapid increase in both phospho and total STAT5 levels upon PRL treatment. SMN mRNA induction is also seen when cells are treated with the small molecule STAT5 activator ATA (28). Conversely, an attenuation of PRL-mediated SMN induction was observed when cells were pretreated with STAT5 RNAi. An earlier report showing in vitro STAT5 conferred SMN induction revealed the induction to be transcriptional in nature (23) (rather than transcript stabilization or altered splicing); in keeping with this, we have found that actinomycin D (a transcription inhibitor) treatment effectively abrogated the SMN induction in NT2 cells (data not shown).

Treatment with different doses of PRL in WT mice next revealed, as with cell culture, a sustained induction of SMN protein in brain and, to a lesser degree, in spinal cord. An increased and optimized PRL dose in SMA mice resulted in a significant and sustained increase in SMN mRNA and protein levels, surpassing that observed in previously identified SMN-inducing agents $(23,30-34)$. Immunohistochemical analysis revealed a significant SMN induction in motor neurons. The second site of profound SMN upregulation appeared to be capillaries and endothelial cells. Although recent work suggests a central nervous system-mediated role in this phenomenon (35), whether the modulation of SMN in 
A

CD-1 mice (brain)

PRL $(\mu \mathrm{g} / \mathrm{kg})$

$\begin{array}{llll}0 & 5.0 & 50 & 500\end{array}$

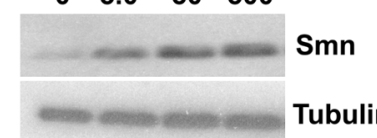

CD-1 mice (spinal cord)

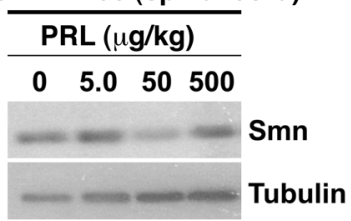

B

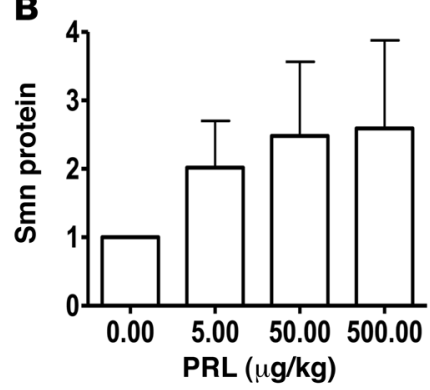

D

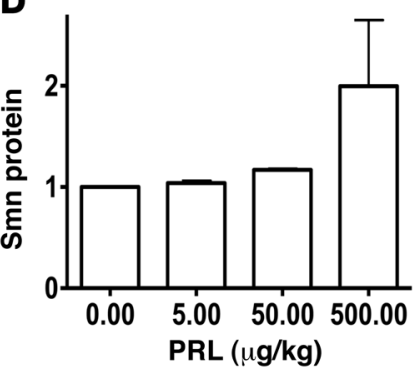

Figure 3

$\mathrm{PRL}$ upregulates Smn protein in WT mice. Male CD-1 WT mice were treated daily with PRL $(5.0,50.0,500.0 \mu \mathrm{g} / \mathrm{kg})$ for 5 days, then sacrificed. Brain and spinal cord tissues were harvested for Western blot analysis. (A) Representative Western blot showing the effect of PRL on Smn protein in brain samples of CD-1 mice treated with saline (control, lane 1) or PRL (lanes 2, 3, and 4, respectively) $(n=3)$. (B) Densitometric quantification of $\mathrm{Smn}$ relative to tubulin is shown for brain samples. (C) Representative Western blot showing the effect of PRL on Smn protein in spinal cord samples of CD-1 mice treated with saline (control, lane 1) or PRL (lanes 2, 3, and 4) $(n=3)$. (D) Densitometric quantification of $S m n$ relative to tubulin is shown for spinal cord samples. Mean + SD. the endothelium also has implications for the peripheral necrosis recently observed in both mouse model of the disease (30) and in patients $(36,37)$ is an open question.

Although the $m S m n^{-/-} ; S M N 2^{+/+}, S M N 2 * \Delta 7^{+/+}$mouse (null for mouse Smn gene rescued with 2 transgenes; human SMN2 and an SMN2 cDNA deleted for exon 7), with a phenotype that closely resembles type I SMA in humans, is one of the most widely used in preclinical assessment of SMA therapeutics (29), interlaboratory comparisons of the impact of drug or other intervention poses a challenge. The sole sure commonality, in addition to its being the same species, is the absence of endogenous mouse Smn and presence of presumably identical human SMN2 and $S M N \triangle 7$ transgenes. Otherwise the genetic background, housing conditions (e.g., temperature, dark/light cycle), and not least, feeding conditions can vary widely as can be seen by survivals ranging from 12 to 17 days. Thus, we find that taking the ratio of median survival of treated to
A

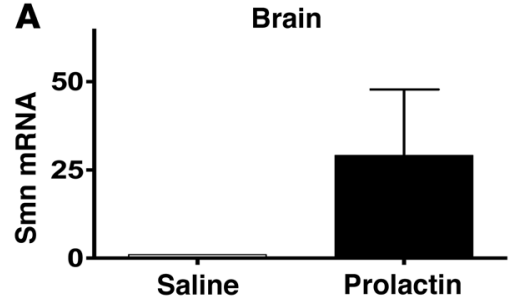

D

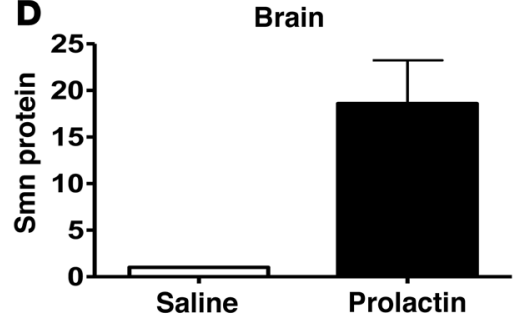

B

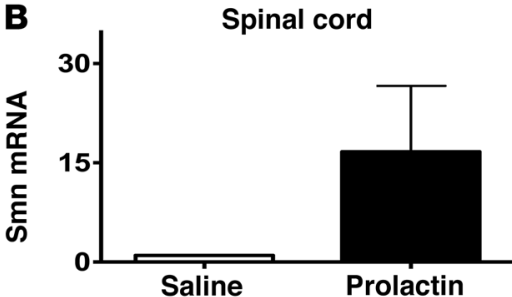

E

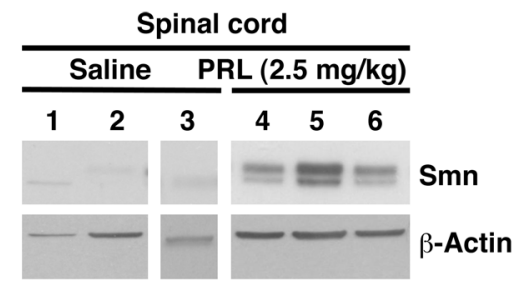

C

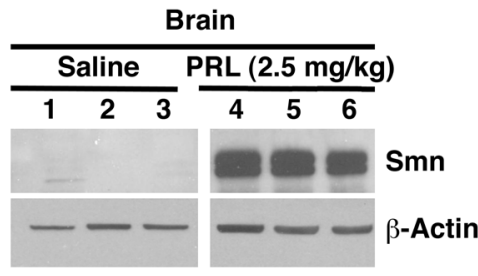

$\mathbf{F}$

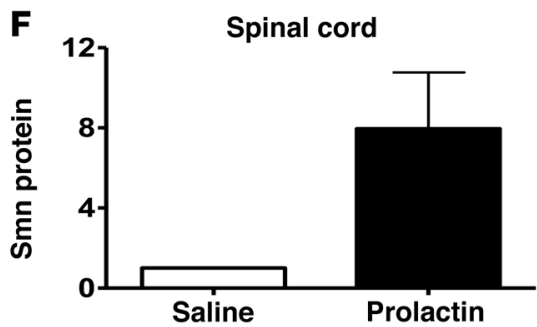

Figure 4

PRL upregulates Smn protein in SMA mouse model. SMA $\Delta 7$ mice were treated daily with saline or PRL (2.5 mg/kg) from P1 for 6 days, then sacrificed at P7. Brain and spinal cord tissues were harvested for RT-PCR and Western blot analysis. (A) Quantification of Smn mRNA relative to $\beta$-actin in brain tissue after PRL treatment (fold induction; the ratio at saline treatment was set as 1). Mean + SD is shown $(n=5)$. (B) Quantification of Smn mRNA relative to $\beta$-actin in spinal cord tissue after PRL treatment (fold induction; the ratio at saline treatment was set as 1). Mean $+\mathrm{SD}$ is shown $(n=5)$. (C) Representative Western blot showing effect of PRL on Smn protein in brain samples of SMA $\Delta 7$ mice treated with saline (control, lanes 1, 2, and 3) or PRL (lanes 4, 5, and 6, respectively) (each lane represents individual animal; all lanes were run on the same gel but were noncontiguous). (D) Densitometric quantification of SMN relative to tubulin is shown for brain samples. Mean + SD. (E) Representative Western blot showing effect of PRL on Smn protein in spinal cord samples of SMA $\Delta 7$ mice treated with saline (control, lanes 1, 2, and 3) or PRL (lanes 4, 5, and 6, respectively) (each lane represents individual animal; all lanes were run on the same gel but were noncontiguous). (F) Densitometric quantification of SMN relative to tubulin is shown for spinal cord samples. Mean + SD. 

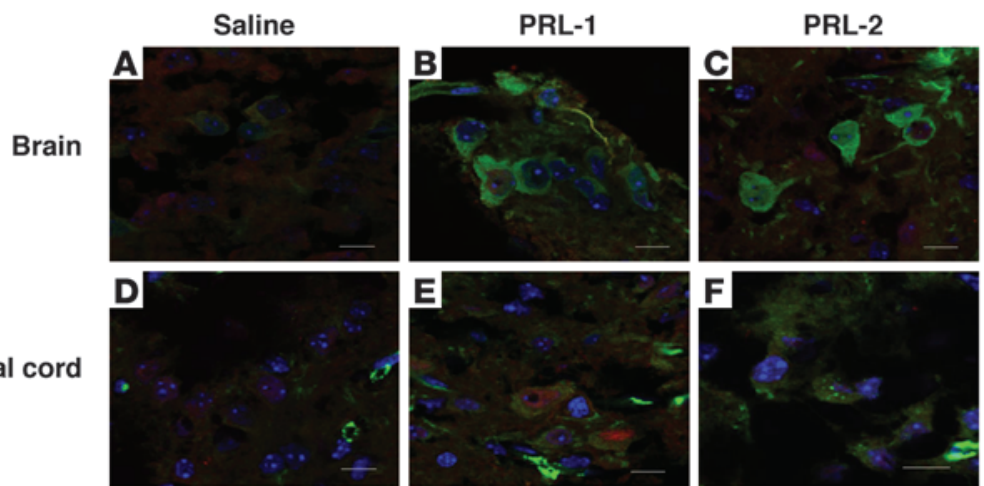

Saline

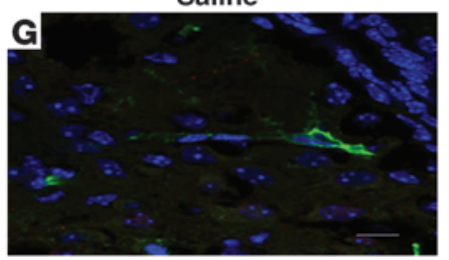

PRL

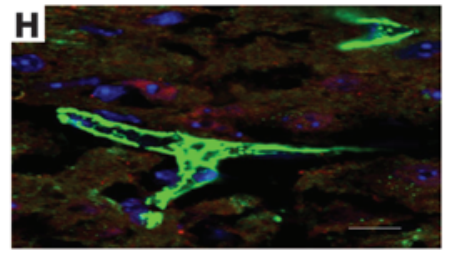

\section{Figure 5}

PRL upregulates Smn protein expression in motor neurons and endothelial cells in SMA mouse model. SMA $\Delta 7$ mice were treated daily with saline or PRL $(2.5 \mathrm{mg} / \mathrm{kg})$ from $\mathrm{P} 1$ for 6 days, then sacrificed at P7. Brain stem and spinal cord tissues were harvested for immunohistochemistry analysis. Representative merged confocal images (Smn/Alexa Fluor 488 [green] + HB9/Alexa Fluor 568 [red; motor neuron marker] plus Hoechst [blue]) for different tissues are shown. Representative confocal images showing effect of PRL on Smn protein expression in brain stem and spinal cord motor neurons samples of SMA $\Delta 7$ mice treated with saline (control, $\mathbf{A}$ and $\mathbf{D}$ ) or PRL ( $\mathbf{B}$ and $\mathbf{C}$, and $\mathbf{E}$ and F), respectively $(n=3)$. Scale bars: $10 \mu \mathrm{M}$. Representative confocal images showing effect of PRL on Smn protein expression in endothelial cells of SMA $\Delta 7$ mice treated with saline (control, G) or PRL $(\mathbf{H})(n=3)$. Scale bars: $10 \mu \mathrm{M}$. nontreated is a useful metric by which to assess effectiveness of a given intervention, irrespective of which laboratory is conducting the analysis. We have achieved a ratio of $21 \mathrm{~d} / 14 \mathrm{~d}$ or 1.6 , a number that compares favorably with the $1.2(19 \mathrm{~d} / 16 \mathrm{~d})$ observed with TSA (albeit P1 PRL initiation versus P5 TSA initiation) and 1.3 $(12.9 \mathrm{~d} / 9.9 \mathrm{~d})$ seen with SAHA $(32,33)$.

In addition to apparently being more effective than the comparatively toxic TSA treatment, the phenomenon of responder and nonresponder mice observed with TSA treatment (33) was not observed with PRL treatment, as the hormone appeared to confer roughly consistent benefit on all treated SMA pups. Another compound that has shown promise for the treatment of SMA is an antisense oligonucleotide that prevents alternative splicing of SMN2 transcript, resulting in more full-length transcript (30). The most encouraging report so far in the field of SMA therapeutics is on the use of self-complementary AAV9 gene therapy with SMN as a payload, which resulted in an extension in longevity of SMA mice from 2 weeks to 250 days plus (38). Similar results have been seen by other groups who used similar gene therapy approaches to treat SMA mice (39-41). However, the clinical introduction of this treatment for SMA must await resolution of the issues of clinical safety, a potential species barrier including immune response, the ability to prepare an adequate quantity of GMP-grade virus, and overall cost (42).

We were struck by the dramatically greater SMN induction observed in SMA mice when compared with WT mice; we also noted that SMN induction in human cell lines surpassed that observed in murine cell lines. The sole sources of SMN protein in the SMA mice are human SMN genes. This prompted an analysis of the putative STAT5-binding sites in murine Smn and human SMN genes. In the original STAT5 SMN paper, promoter sequence analysis of both murine and human $S M N$ genes showed 2 conserved Stat5-binding sites (TTCNNNGAA/TTCNNNTAA) in the murine SMN promoter (NCBI AF027668) but none in the human SMN2 promoter (NCBI AF027688). Three similar CTCNNNTAA elements were detected uniquely in the SMN2 promoter (-413 to $-409 \mathrm{bp},-2338$ to $-2330 \mathrm{bp}$, and -3881 to -3873 bp downstream of the SMN2 start codon [+1]; ref. 23). However, our further analysis using the less stringent online database known as DECODE (the Champion ChiP Transcription Factor Search Portal based on SABiosciences' proprietary database; http://www.sabiosciences.com/chipqpcrsearch.php?app=TFBS)
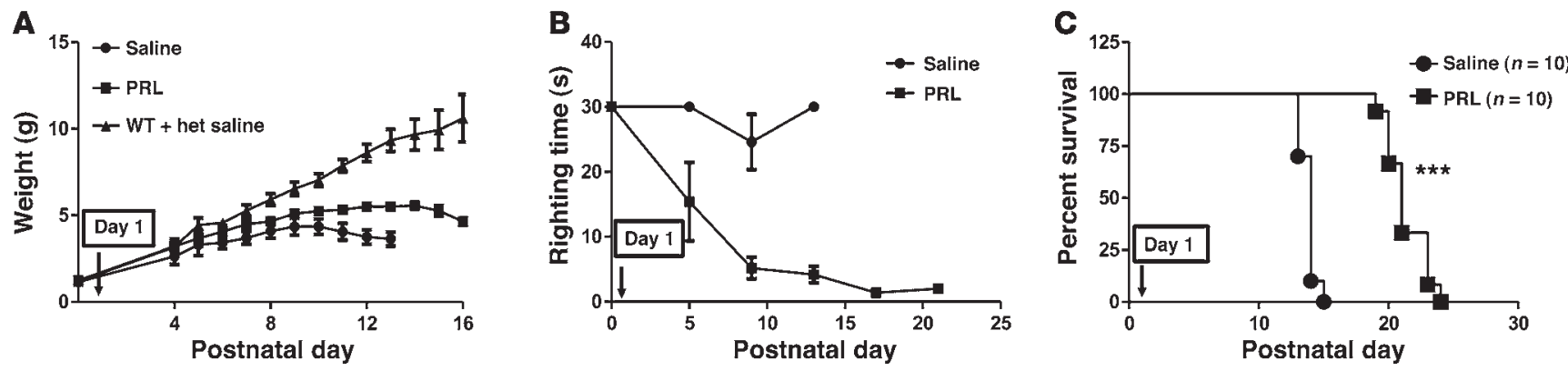

Figure 6

PRL ameliorates disease phenotype in SMA mouse model. SMA $\Delta 7$ mice were treated daily with i.p. injections of PRL (2.5 mg/kg) from P1 onward. (A) Weights of SMA $\triangle 7$ mice treated with PRL (squares) or saline (circles) $(n=5)$ and weights for heterozygous mice treated with saline (triangles) are also shown for comparison. Mean \pm SD. (B) Righting times of SMA $\triangle 7$ mice treated with PRL (squares) or saline (circles) $(n=5)$. Mean \pm SD. (C) Kaplan-Meier survival curves of SMA $\Delta 7$ mice treated with PRL (squares) or vehicle (circles) $(n=10)$; ${ }^{* * *} P<0.0001$, log-rank test. 
A
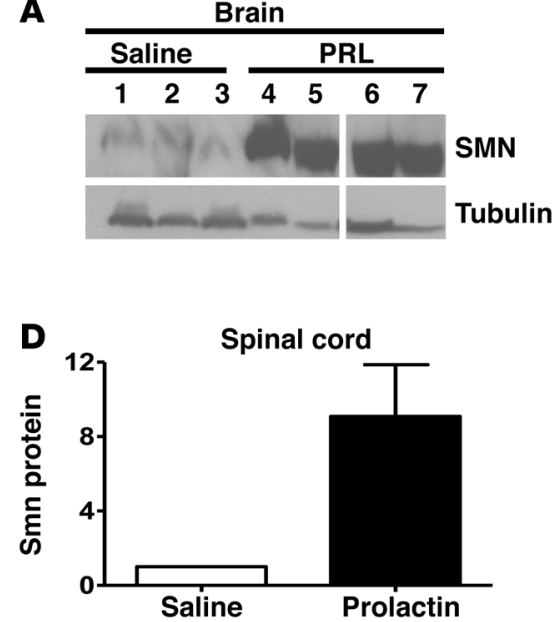

B

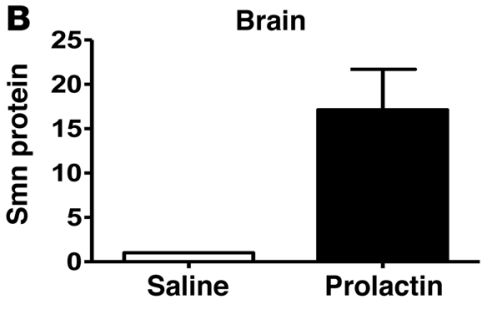

E

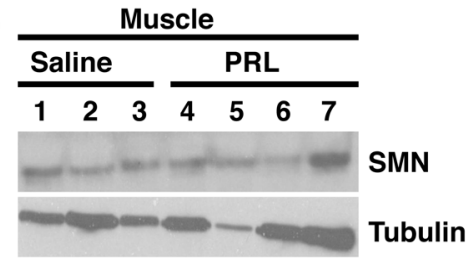

C
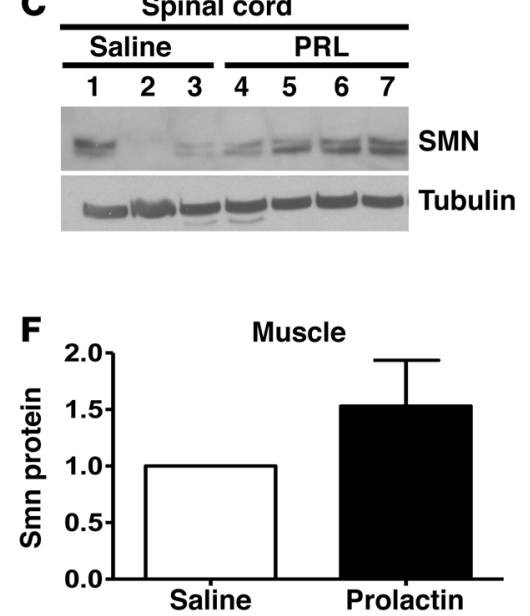

Figure 7

Smn protein level is upregulated in SMA $\Delta 7$ mice until time of death. SMA $\Delta 7$ mice were treated daily with PRL $(2.5 \mathrm{mg} / \mathrm{kg})$ from P1 onward. Brain, spinal cord, and muscle tissues were harvested upon death for Western blot analysis. (A) Representative Western blot showing the effect of PRL on Smn protein in brain samples of SMA $\Delta 7$ mice treated with saline (control, lane 1, 2, and 3) or PRL (lane 4, 5, 6, and 7) (each lane represents individual animal; all lanes were run on the same gel but were noncontiguous). (B) Densitometric quantification of SMN relative to tubulin is shown for brain samples. Mean \pm SD. (C) Representative Western blot showing effect of PRL on Smn protein in spinal cord samples of SMA $\Delta 7$ mice treated with saline (control, lanes 1, 2, and 3) or PRL (lanes 4, 5, 6, and 7, respectively) (each lane represents individual animal). (D) Densitometric quantification of SMN relative to tubulin is shown for spinal cord samples. Mean \pm SD. (E) Representative Western blot showing the effect of PRL on Smn protein in muscle samples of SMA $\Delta 7$ mice treated with saline (control, lanes 1, 2, and 3) or PRL (lanes 4, 5, 6, and 7) (each lane represents individual animal). (F) Densitometric quantification of SMN relative to tubulin is shown for muscle samples. Mean \pm SD.

revealed a total of twelve STAT5-binding sites for the human SMN2 and none for mouse Smn promoter. We believe that this difference may account for the profound SMN induction we see when SMN2 is the source of SMN protein.

PRL treatment in SMA mice has revealed a significant in vivo induction of SMN protein, which correlates with an overall improvement in the phenotype of the disease. PRL treatment attenuated the weight loss and improved motor neuron function considerably; it also resulted in an approximately $70 \%$ increase in life span in SMA mice. However, the degree of SMN induction, greater than that observed in heterozygote mice that have a normal life span (Supplemental Figures 4 and 5), is at odds with the significant but in contrast comparatively modest improvement in longevity. Four obvious sources of this disconnect are as follows: (a) notwithstanding the P1 inception of treatment, a delay in SMN induction in the target motor neuron; (b) a role for SMN in other neuronal cells besides motor neurons refractory to PRLmediated induction; (c) a role for SMN in other tissues refractory to PRL-mediated induction; and (d) inconsistent SMN induction. Given the small size of P1 and P2 pups, there can be technical challenges in ensuring complete administration of the total PRL dose in the first few days of life. However, the recent AAV 9 SMN gene therapy rescue of the same mouse model work has shown that motor neuron transduction as late as 5 days still results in survival extension to 40 days, suggesting that a failure to induce SMN

\section{Figure 8}

Proposed model for PRL-mediated induction of SMN in motor neurons. PRL treatment causes phosphorylation and activation of STAT5, pathway which results in transcriptional upregulation of $S M N$ gene, resulting in an increase in SMN mRNA levels. This ultimately increases SMN protein expression. in other neuronal cells or in tissues other than the motor neuron may be the more likely source of the mortality (38). In this regard, the recent observation of significant cardiac pathology in this mouse model (43-45) combined with the lack of SMN induction we observed in the myocardium tissue (Supplemental Figure 2) suggest that cardiac failure may underlie the early mortality. However, it has been shown that induction of SMN in heart tissue itself does not rescue SMA mice, whereas neuronal-specific transgenic

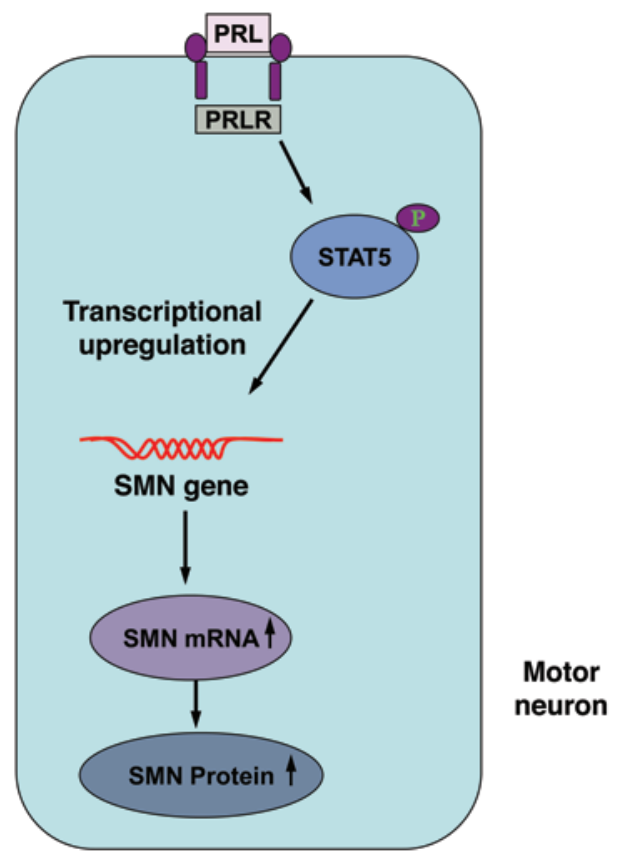


expression of SMN ameliorates disease phenotype with an increase in the survival of SMA mice (46). It may be that SMN levels higher than those induced by $\operatorname{Prl}$ are uniquely required in the neurons that innervate heart tissue.

Clinical experience with PRL is limited, although a recent study has demonstrated the safe and effective use of recombinant PRL for mothers with lactation insufficiency (47). Moreover, PRL has been shown to regulate oligodendrocyte precursor proliferation and mimic the regenerative effects of pregnancy. PRL's striking ability to repair demyelination identifies it as a potential therapeutic agent in multiple sclerosis (48). There could be some potential side effects of higher levels of PRL. A condition called hyperprolactinaemia (resulting from higher levels of blood PRL) can lead to hypoestrogenism, which may result in infertility and osteoporosis (49). Higher levels of PRL also decrease dopamine release; therefore, some antipsychotic and antidepressant drugs can elevate PRL levels as well.

Our results demonstrate a clear promise for PRL use in clinical trial studies: amelioration of disease phenotype in a mouse model, $\mathrm{BBB}$ penetration, and safe and FDA-approved status. Recent work has shown that the maximum treatment benefit for murine SMA is contingent upon early timing of SMN gene therapy. An early diagnosis followed by a prompt initiation of PRL treatment in SMA newborns may therefore be key to an optimal outcome in humans. We hope that SMA type II and III patients will also benefit from PRL treatment, as we hope an increase in SMN levels upon treatment will ameliorate disease progression and will improve the function of the remaining motor neurons. It will also be interesting to combine the effect of PRL with SMN2 transcript stabilizers (50) and/or neuroprotective compounds such as Y-27632 (Rho kinase inhibitor) (51).

Presently there is no cure for SMA. This study provides a good mechanistic insight into how SMN protein is regulated through PRL via the STAT5 pathway (Figure 8) and its effect on the phenotype of the disease as well its potential for future therapeutic use for the treatment of SMA.

\section{Methods}

Animals. All experiments were performed in accordance with Animal Care and Veterinary Services (ACVS), University of Ottawa, legislation. All protocols were approved by Animal Care and Veterinary Services and Ethics of the University of Ottawa and all experiments were carried out in accordance with the Canadian Institute of Health Research (CIHR) Guidebook. CD-1 mice were obtained from Charles River Laboratory. The original breeding pair of heterozygous SMA $\Delta 7$ mice $\left(\mathrm{mSmn}^{+/-} ; \mathrm{SMN2}^{+/+}\right.$, $S M N 2 * \Delta 7^{+/+}$) on the FVB background were provided by the Jackson Laboratory. The animals were maintained in an air-conditioned, ventilated animal facility. Survival, righting time, and weight were monitored daily as described by Avila et al. (33).

PRL administration. The mouse or human recombinant PRL was diluted in PBS and administered through i.p. injection using a 30-gauge needle. Control animals received equal volumes of vehicle alone. SMA $\Delta 7$ mice were genotyped at P0, and PRL treatment was started from P1. Animals were sacrificed within 24 hours of final PRL dose.

Reagents. PRL was purchased from Cedarlane Labs, and ATA was from Sigma-Aldrich. Nonsilencing siRNA control and STAT5 siRNA (\#6275) were supplied by QIAGEN and Cell Signaling, respectively. The antibodies used in this study were SMN/Smn (BD Transduction Laboratories), PRLR (Abcam), actin (Abcam), tubulin (Abcam), phospho-STAT5 (Cell Signaling), total STAT5 (Cell Signaling), and HB9 (Abcam).
Primer sequences. For q-PCR, sequences were as follows: SMN, human (amplifies full-length transcript only) forward: 5'-GCTATCATACTGATACTGGCTATTATATGGGTTTTT-3'; reverse: 5'-CTATAACGCTTCACATTCCAGATCTG-3'; actin, forward: 5'-CTGGAACGGTGAAGGTGACA-3'; reverse: 5'-AAGGGACTTCCTGTAACAATGCA-3'.

Genotyping was performed as previously described by Avila et al. (33) using the following primers: $m S m n$ WT, forward: 5'-TCTGTGTTCGTGCGTGGTGACTTT-3'; reverse 1877: 5'-CCCACCACCTAAGAAAGCCTCAAT-3'; Lac Z, forward: 5'-CCAACTTAATCGCCTTGCAGCACA-3'; reverse: 5'-AAGCGAGTGGCAACATGGAAATCG 3'; human SMN2 transgene, forward: 5'-CAAACACCTGGTATGGTCAGTC-3'; reverse: 5'-GCACCACTGCACAACAGCCTG-3'. Product sizes were as follows: $m S M N$ : $372 \mathrm{bp}$; Lac Z: 626 bp; SMN2 transgene: 250 bp (Supplemental Figure 3).

Cell culture and drug treatment conditions. Human NT2 or MN-1 cells were maintained in standard conditions $\left(37^{\circ} \mathrm{C}\right.$ in a $5 \% \mathrm{CO}_{2}$ humidified atmosphere) in DMEM supplemented with $10 \%$ FCS, $1 \%$ antibiotics (100 units $/ \mathrm{ml}$ penicillin- streptomycin), and $2 \mathrm{mM}$ glutamate.

NT2 or MN-1 cells were seeded in 12 -well plates $\left(2.5 \times 10^{5}\right.$ cells/well $)$ and treated beginning 24 hours later with PRL $(25,50,75$, and $100 \mathrm{ng} / \mathrm{ml})$ for 48 hours. For time-course experiment, NT2 cells were seeded in 12-well plates $\left(2.5 \times 10^{5}\right.$ cells/well $)$ and treated beginning 24 hours later with PRL (50 ng/ml) for up to 1 hour. For ATA treatment, NT2 was seeded in 12 -well plates $\left(2.5 \times 10^{5}\right.$ cells/well $)$ and treated beginning 24 hours later with ATA $(125 \mu \mathrm{M})$ for 24 hours.

Transfection. For siRNA transfections, NT2 cells were seeded in 12-well plates $\left(2.0 \times 10^{5}\right.$ cells/well $)$ and transfected on the following day in serumfree DMEM with STAT5 siRNA $(50 \mathrm{nM})$ or nonsilencing control siRNA $(50 \mathrm{nM})$, using LipofectAMINE 2000 transfection reagent for 48 hours.

Quantitative RT-PCR. Total RNA was isolated according to the protocol provided by the manufacturer using the RNeasy kit (QIAGEN). For quantitative RT-PCR, cDNA was reverse transcribed from isolated RNA with oligo $\mathrm{dT}_{18}$ primer using the First-Strand cDNA Synthesis Kit (GE Healthcare) following the manufacturer's instructions. The synthesized cDNA template was used for quantitative PCR employing the QuantiTect SYBR Green PCR Kit (QIAGEN) and analyzed on an ABI Prism 7000 sequence detection system using the ABI Prism 7000 SDS Software. Quantitative PCR was carried out to detect $\mathrm{SMN}$ and actin genes using the primers listed above.

Western blot analysis. Cells were washed 2 times with $1 \mathrm{ml} \mathrm{PBS} \mathrm{(1 \times )} \mathrm{and} \mathrm{lysed}$ in $75 \mu \mathrm{l}$ RIPA buffer containing $10 \mathrm{mg} / \mathrm{ml}$ each of aprotinin, PMSF, and leupeptin (all from Sigma-Aldrich), $5 \mathrm{mM} \beta$-glycerophosphate, $50 \mathrm{mM} \mathrm{NaF}$, and $0.2 \mu \mathrm{M}$ sodium orthovanadate for 30 minutes at $4^{\circ} \mathrm{C}$, followed by centrifugation at $13000 \mathrm{~g}$ for 15 minutes; supernatants were then collected and kept frozen at $-20^{\circ} \mathrm{C}$. Tissue samples were homogenized in $1 \mathrm{ml} \mathrm{RIPA}(10 \mathrm{mg} / \mathrm{ml}$ each of aprotinin, PMSF, and leupeptin) and then sonicated for 15 seconds. Total protein concentrations were determined by Bradford protein assay using a Bio-Rad protein assay kit. For Western blot analysis, protein samples were separated by $10 \%$ SDS-PAGE. Proteins were subsequently transferred onto nitrocellulose membrane and incubated in blocking solution (PBS, $5 \%$ nonfat milk, $0.2 \%$ Tween-20) for 1 hour at room temperature followed by overnight incubation with primary antibody at $4^{\circ} \mathrm{C}$ at the dilution prescribed by the manufacturer. Membranes were washed with PBS-T (PBS, and $0.2 \%$ Tween-20) 3 times followed by incubation with secondary antibody (anti-mouse or rabbit; Cell Signaling) for 1 hour at room temperature. Antibody complexes were visualized by autoradiography using the ECL Plus and ECL Western Blotting detection systems (GE Healthcare). Quantification was performed by scanning the autoradiographs, and signal intensities were determined by densitometry analysis using the Odyssey v1.1 program.

Immunofluorescence staining and confocal microscopy. Brain stems and spinal cords were briefly rinsed in PBS, fixed for 6 hours in $2 \%$ paraformaldehyde in PBS, and then transferred for cryopreservation 
into $30 \%$ sucrose/PBS prior to the making of the cryostat blocks. $20-\mu \mathrm{m}$ sections were obtained with a cryostat, collected onto positively charged slides, and air dried for 1 hour at room temperature. The slides were then incubated for 30 minutes with $0.2 \%$ Triton X-100/PBS, briefly rinsed with PBS, and then incubated with $10 \%$ normal goat serum in PBS. Blocking solution was discarded, and the slides were then incubated overnight at $4{ }^{\circ} \mathrm{C}$ with the mouse anti-SMN antibody diluted in PBS at 1:1000 (BD Biosciences - Transduction Laboratories) and the rabbit anti-HB9 neuronal marker diluted in PBS at 1:1000 (Abcam). After incubation with the primary antibodies, the slides were rinsed 3 times for 10 minutes with PBS and then incubated for 1 hour at room temperature with goat anti-mouse Alexa Fluor 488 and goat anti-rabbit Alexa Fluor 568 (Invitrogen) diluted at 1:1000 in PBS. The slides were then rinsed 3 times for 10 minutes with PBS, counterstained for 5 minutes with Hoechst 33342 (Invitrogen), diluted at $10 \mu \mathrm{g} / \mathrm{ml}$ in PBS, and mounted with Dako Fluorescent Mounting Medium. Confocal microscopy was performed with an Olympus FluoView FV1000 confocal microscope. Confocal microscope settings remained constant for each of the channels imaged. Channels were acquired in a sequential mode; the laser output was set at 5\% and the confocal aperture was set at $176 \mu \mathrm{m}$.

Statistics. GraphPad Prism software package (version 5; GraphPad Software) was used for the Kaplan-Meier survival analysis. The log-rank test was used, and survival curves were considered significantly different at

1. Pearn J. Incidence, prevalence, and gene frequency studies of chronic childhood spinal muscular atrophy. J Med Genet. 1978;15(6):409-413.

2. Emery AE. Population frequencies of inherited neuromuscular diseases--a world survey. Neuromuscul Disord. 1991;1(1):19-29.

3. Lefebvre $S$, et al. Identification and characterization of a spinal muscular atrophy-determining gene. Cell. 1995;80(1):155-165.

4. Friesen WJ, Dreyfuss G. Specific sequences of the $\mathrm{Sm}$ and Sm-like (Lsm) proteins mediate their interaction with the spinal muscular atrophy disease gene product (SMN).J Biol Chem. 2000;275(34):26370-26375.

5. Buhler D, Raker V, Luhrmann R, Fischer U. Essential role for the tudor domain of SMN in spliceosomal U snRNP assembly: implications for spinal muscular atrophy. Hum Mol Genet. 1999;8(13):2351-2357.

6. Jones KW, et al. Direct interaction of the spinal muscular atrophy disease protein SMN with the small nucleolar RNA-associated protein fibrillarin. J Biol Chem . 2001;276(42):38645-38651.

7. Meister G, Buhler D, Laggerbauer B, Zobawa M, Lottspeich F, Fischer U. Characterization of a nuclear 20S complex containing the survival of motor neurons (SMN) protein and a specific subset of spliceosomal Sm proteins. Hum Mol Genet. 2000;9(13):1977-1986.

8. Mourelatos Z, Abel L, Yong J, Kataoka N, Dreyfuss G. SMN interacts with a novel family of hnRNP and spliceosomal proteins. EMBOJ. 2001;20(19):5443-5452.

9. Narayanan U, Achsel T, Luhrmann R, Matera AG. Coupled in vitro import of $U$ snRNPs and SMN, the spinal muscular atrophy protein. Mol Cell. 2004;16(2):223-234.

10. Pellizzoni L, Baccon J, Charroux B, Dreyfuss G. The survival of motor neurons (SMN) protein interacts with the snoRNP proteins fibrillarin and GAR1. Curr Biol. 2001;11(14):1079-1088.

11. Pellizzoni L, Charroux B, Dreyfuss G. SMN mutants of spinal muscular atrophy patients are defective in binding to snRNP proteins. Proc Natl Acad Sci U S A. 1999;96(20):11167-11172.

12. Pellizzoni L, Charroux B, Rappsilber J, Mann M, Dreyfuss G. A functional interaction between the survival motor neuron complex and RNA polymerase II. J Cell Biol. 2001;152(1):75-85. 2008;116(3):223-234.

$P<0.0001$. Data in figures (histograms, points on graphs) are mean values with the SD shown as error bars.

\section{Acknowledgments}

We thank Nehal Thakor, Fahad Shamim, and Djan Brun for technical help and insightful discussion; special thanks to Henry Friesen for important preliminary work. We also thank all those at the Animal Care and Veterinary Service at the University of Ottawa who have been so helpful. M. Holcik is a CHEO Volunteer Association endowed scholar. This work was supported by operating grants from the National Science and Engineering Research Council (NSERC) (to M. Holcik) and from Tori's Buddies, CML Healthcare, FightSMA, the SMA Foundation, the Canadian Gene Cure Foundation, and Families of SMA (to A. MacKenzie). F.A. Molina was supported by the University of Granada GREIB program.

Received for publication January 2, 2011, and accepted in revised form June 1, 2011.

Address correspondence to: Alex MacKenzie, Children's Hospital of Eastern Ontario Research Institute, Apoptosis Research Center, 401 Smyth Road, Ottawa, Ontario K1H 8L1, Canada. Phone: 613.737.2772; Fax: 613.738.4833; E-mail: mackenzie@cheo.on.ca.

13. Fischer U, Liu Q, Dreyfuss G. The SMN-SIP1 complex has an essential role in spliceosomal snRNP biogenesis. Cell. 1997;90(6):1023-1029.

14. Brzustowicz LM, et al. Genetic mapping of chronic childhood-onset spinal muscular atrophy to chromosome 5q11.2-13.3. Nature. 1990;344(6266):540-541.

15. Melki J, et al. Gene for chronic proximal spinal muscular atrophies maps to chromosome $5 \mathrm{q}$. Nature. 1990;344(6268):767-768.

16. Lorson CL, Hahnen E, Androphy EJ, Wirth B. A single nucleotide in the SMN gene regulates splicing and is responsible for spinal muscular atrophy. Proc Natl Acad Sci U S A. 1999;96(11):6307-6311.

17. Monani UR, et al. A single nucleotide difference that alters splicing patterns distinguishes the SMA gene SMN1 from the copy gene SMN2. Hum Mol Genet. 1999;8(7):1177-1183.

18. Schrank B, et al. Inactivation of the survival motor neuron gene, a candidate gene for human spinal muscular atrophy, leads to massive cell death in early mouse embryos. Proc Natl Acad Sci U S A. 1997;94(18):9920-9925.

19. Simic G. Pathogenesis of proximal autosomal recessive spinal muscular atrophy. Acta Neuropathol.

20. Kariya S, Mauricio R, Dai Y, Monani UR. The neuroprotective factor Wld(s) fails to mitigate distal axonal and neuromuscular junction (NMJ) defects in mouse models of spinal muscular atrophy. Neurosci Lett. 2009;449(3):246-251.

21. Campbell L, Potter A, Ignatius J, Dubowitz V, Davies K. Genomic variation and gene conversion in spinal muscular atrophy: implications for disease process and clinical phenotype. Am J Hum Genet. 1997;61(1):40-50.

22. McAndrew PE, et al. Identification of proximal spinal muscular atrophy carriers and patients by analysis of SMNT and SMNC gene copy number. Am J Hum Genet. 1997;60(6):1411-1422.

23. Ting CH, Lin CW, Wen SL, Hsieh-Li HM, Li H. Stat5 constitutive activation rescues defects in spinal muscular atrophy. Hum Mol Genet. 2007;16(5):499-514.

24. Markham K, Schuurmans C, Weiss S. STAT5A/B activity is required in the developing forebrain and spinal cord. Mol Cell Neurosci. 2007;35(2):272-282.

25. Goffin V, et al. From the molecular biology of prolactin and its receptor to the lessons learned from knockout mice models. Genet Anal.
1999;15(3-5):189-201.

26. Olazabal I, Munoz J, Ogueta S, Obregon E, Garcia-Ruiz JP. Prolactin (PRL)-PRL receptor system increases cell proliferation involving JNK (c-Jun amino terminal kinase) and AP-1 activation: inhibition by glucocorticoids. Mol Endocrinol. 2000;14(4):564-575.

27. Royster M, Driscoll P, Kelly PA, Freemark M. The prolactin receptor in the fetal rat: cellular localization of messenger ribonucleic acid, immunoreactive protein, and ligand-binding activity and induction of expression in late gestation. Endocrinology. 1995;136(9):3892-3900.

28. Rui H, et al. Activation of the Jak2-Stat5 signaling pathway in Nb2 lymphoma cells by an anti-apoptotic agent, aurintricarboxylic acid. J Biol Chem. 1998;273(1):28-32.

29. Le TT, et al. SMNDelta7, the major product of the centromeric survival motor neuron (SMN2) gene, extends survival in mice with spinal muscular atrophy and associates with full-length SMN. Hum Mol Genet. 2005;14(6):845-857.

30. Williams JH, Schray RC, Patterson CA, Ayitey SO, Tallent MK, Lutz GJ. Oligonucleotide-mediated survival of motor neuron protein expression in CNS improves phenotype in a mouse model of spinal muscular atrophy. J Neurosci. 2009;29(24):7633-7638.

31. Wirth B, Brichta L, Hahnen E. Spinal muscular atrophy and therapeutic prospects. Prog Mol Subcell Biol. 2006;44:109-132.

32. Riessland M, et al. SAHA ameliorates the SMA phenotype in two mouse models for spinal muscular atrophy. Hum Mol Genet. 2010;19(8):1492-1506

33. Avila AM, et al. Trichostatin A increases SMN expression and survival in a mouse model of spinal muscular atrophy. J Clin Invest. 2007;117(3):659-671.

34. Tsai LK, Tsai MS, Ting CH, Li H. Multiple therapeutic effects of valproic acid in spinal muscular atrophy model mice. J Mol Med. 2008;86(11):1243-1254.

35. Hua Y, et al. Antisense correction of SMN2 splicing in the CNS rescues necrosis in a type III SMA mouse model. Genes Dev. 2010;24(15):1634-1644.

36. Rudnik-Schoneborn S, Vogelgesang S, Armbrust S, Graul-Neumann L, Fusch C, Zerres K. Digital necroses and vascular thrombosis in severe spinal muscular atrophy. Muscle Nerve. 2010;42(1):144-147.

37. Araujo Ade Q, Araujo M, Swoboda KJ. Vascular perfusion abnormalities in infants with spinal 
muscular atrophy. J Pediatr. 2009;155(2):292-294.

38. Foust KD, et al. Rescue of the spinal muscular atrophy phenotype in a mouse model by early postnatal delivery of SMN. Nat Biotechnol. 2010;28(3):271-274.

39. Passini MA, et al. CNS-targeted gene therapy improves survival and motor function in a mouse model of spinal muscular atrophy. J Clin Invest. 2010;120(4):1253-1264.

40. Valori CF, et al. Systemic delivery of scAAV9 expressing SMN prolongs survival in a model of spinal muscular atrophy. Sci Transl Med. 2010;2(35):35ra42.

41. Dominguez E, et al. Intravenous scAAV9 delivery of a codon-optimized SMN1 sequence rescues SMA mice. Hum Mol Genet. 2011;20(4):681-693.

42. MacKenzie A. Genetic therapy for spinal muscular atrophy. Nat Biotechnol. 2010;28(3):235-237.
43. Bevan AK, et al. Early heart failure in the SMNDelta7 model of spinal muscular atrophy and correction by postnatal scAAV9-SMN delivery. Hum Mol Genet. 2010;19(20):3895-3905.

44. Heier CR, Satta R, Lutz C, DiDonato CJ. Arrhythmia and cardiac defects are a feature of spinal muscular atrophy model mice. Hum Mol Genet. 2010;19(20):3906-3918.

45. Shababi M, Habibi J, Yang HT, Vale SM, Sewell WA, Lorson CL. Cardiac defects contribute to the pathology of spinal muscular atrophy models. Hum Mol Genet. 2010;19(20):4059-4071.

46. Gavrilina TO, et al. Neuronal SMN expression corrects spinal muscular atrophy in severe SMA mice while muscle-specific SMN expression has no phenotypic effect. Hum Mol Genet. 2008;17(8):1063-1075.
47. Powe CE, et al. Recombinant human prolactin for the treatment of lactation insufficiency. Clin Endocrinol (Oxf). 2010;73(5):645-653.

48. Gregg C, et al. White matter plasticity and enhanced remyelination in the maternal CNS. J Neurosci. 2007;27(8):1812-1823.

49. Delemer B. Prolactinomas: diagnosis and treatment. Presse Med. 2009;38(1):117-124.

50. Farooq F, Balabanian S, Liu X, Holcik M, MacKenzie A. p38 Mitogen-activated protein kinase stabilizes SMN mRNA through RNA binding protein HuR. Hum Mol Genet. 2009;18(21):4035-4045.

51. Bowerman M, Beauvais A, Anderson CL, Kothary R. Rho-kinase inactivation prolongs survival of an intermediate SMA mouse model. Hum Mol Genet. 2010;19(8):1468-1478. 